\title{
On the reliable solution of contact problems in engineering design
}

\author{
NAGI ELABBASI ${ }^{1}$, JUNG-WUK HONG ${ }^{2}$, and KLAUS-JÜRGEN BATHE ${ }^{2, *}$ \\ ${ }^{1}$ ADINA R\&D, Inc., 71 Elton Avenue, Watertown, MA 02472, USA; ${ }^{2}$ Massachusetts Institute of Technology, 77 \\ Massachusetts Avenue, Cambridge, MA 02139, USA \\ *Author for correspondence (E-mail: kjb@mit.edu; Phone: +1-617-253-6645; Fax: +1-617-253-2275)
}

Received 2 June 2003; accepted in revised form 6 December 2003

\begin{abstract}
In this paper we examine briefly the reliability of solution needed for the accurate and effective analysis of engineering design problems involving contact conditions. A general finite element formulation for treating the frictional contact problem using constraint functions is first summarized. Then we address general reliability issues and those related to the selection of appropriate elements that provide optimal performance. These elements of course do not lock and would provide the best solution an analyst can expect when simulating a design problem. Reliability issues specific to the contact formulation are also presented. A promising procedure to increase the reliability of an analysis is the method of finite spheres. The method does not require a mesh and in particular can be used with a finite element discretization as described in the paper. Finally, the results of several illustrative analysis problems are given.
\end{abstract}

Key words: contact, reliability, finite element solution, finite spheres, design

\section{Introduction}

Finite element methods are used extensively in many engineering applications to analyze new and existing designs. The complexity of many design problems, as well as the scarcity of test data, require analysis solutions and therefore that the most accurate and reliable numerical methods for analysis be employed. This includes using the appropriate finite element formulations, using the appropriate elements, and using an appropriate contact formulation (Bathe, 1996).

The finite element formulation selected must be suitable for the problem to be analyzed. For example, only implicit analysis should be used for low and medium speed simulations. The selected elements should be stable and effective. If optimal elements are used, reliable results are obtained for any geometry and boundary conditions. Of course, reliable elements will not give unstable solutions, will not lock and are not based on "numerical factors". Finally, the contact formulation should be reliable. It should satisfy the appropriate physical and mathematical conditions, should not ill-condition the stiffness matrices, and-like the elements used-should possess the optimal convergence rate (Bathe 1996, 2001a; El-Abbasi and Bathe, 2001).

In this paper, we briefly present a finite element formulation for contact problems based on the constraint function method. We then address the different reliability aspects mentioned above, and finally, we present some state-of-the-art illustrative numerical results that were obtained using reliable finite element methods.

\section{Theory}

Consider a system consisting of two bodies in contact (Figure 1). The contact kinematics dictate that for any admissible displacement $\mathbf{v}$, there is no inter-penetration between the bodies, and the 


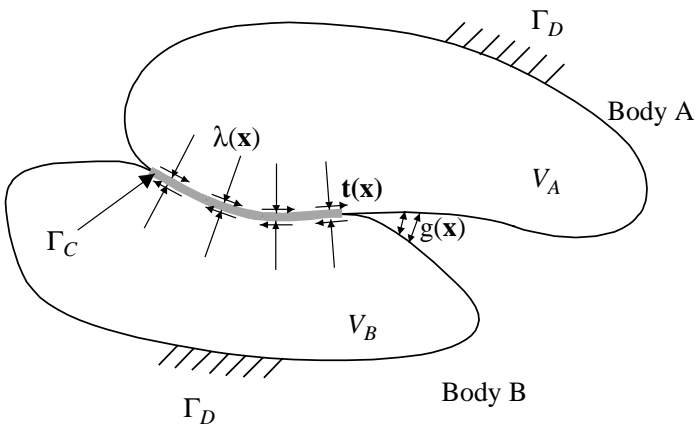

Figure 1. Two bodies in contact.

contact pressure $\lambda$ can only be zero or positive. These normal contact conditions can be represented as

$$
g \geq 0, \quad \lambda \geq 0, \quad g \lambda=0
$$

where $g$ is the gap which can be defined as

$$
g=\left[\mathbf{x}_{2}-\mathbf{x}_{1}\right] \cdot \mathbf{n}
$$

where $\mathbf{x}_{1}$ and $\mathbf{x}_{2}$ are appropriately selected points on the surfaces of bodies $A$ and $B$, and $\mathbf{N}$ is a unit normal vector.

For the tangential contact conditions, we assume that once contact is established, a relative contact tangential motion is possible only if the magnitude of the tangential traction $\boldsymbol{t}$ is sufficiently large to overcome the frictional resistance at the contact surface. We define the nondimensional frictional variable $\tau$ as

$$
\tau= \begin{cases}\frac{|t|}{\zeta} & \text { if } \zeta>0 \\ 0 & \text { otherwise }\end{cases}
$$

where the scalar $\zeta$ represents the frictional resistance. In the case of Coulomb friction,

$$
\zeta=\mu \lambda
$$

The relative tangential velocity between the contacting bodies can be defined as

$$
v=\left[\mathbf{v}_{2}-\mathbf{v}_{1}\right] \cdot \mathbf{s}
$$

where $\mathbf{s}$ is the unit vector in the slipping direction. The stick/slip condition can be expressed as

$$
\tau \leq 1, \quad \tau<1 \Rightarrow v=0, \quad \tau=1 \Rightarrow \text { slip : } v \neq 0
$$

The variational formulation of the frictional contact problem involving $N$ contacting bodies can be expressed as (Bathe, 1996)

$$
\begin{aligned}
& \sum_{I=1}^{N} \int_{{ }^{0} V^{I}} \mathbf{S} \cdot \delta \boldsymbol{\varepsilon} \mathrm{d}^{0} V^{I}+\sum \int_{\Gamma_{\mathrm{C}}}\left(\lambda \mathbf{n}^{J}+\mathbf{t}^{J}\right) \cdot \Delta \delta \mathbf{u}^{I J} \mathrm{~d} \Gamma_{\mathrm{C}}=\sum_{I=1}^{N} \int_{{ }^{0} V^{I}} \rho\left(\mathbf{f}^{B}-\ddot{\mathbf{u}}\right) \cdot \delta \mathbf{u} \mathrm{d}^{0} V^{I} \\
& \quad+\sum_{I=1}^{N} \int_{\partial^{0} V^{I}} \mathbf{f}^{S} \cdot \delta \mathbf{u} \mathrm{d} \partial^{0} V^{I}
\end{aligned}
$$


where

$$
\Delta \delta \mathbf{u}^{I J}=\delta \mathbf{u}^{J}-\delta \mathbf{u}^{I}
$$

denotes the virtual displacement of body $J$ relative to body $I$. The contact terms in Eq. (7) are evaluated over the yet unknown contact area $\Gamma_{\mathrm{C}}$. A detailed formulation, also accounting for incompressibility (using the $\mathrm{u} / \mathrm{p}$ formulation) and thermal effects, can be found in Pantuso et al. (2000).

Using the constraint function method (Bathe, 1996), the normal contact inequality condition can be replaced by

$$
w_{n}(g, \lambda)=0
$$

where $w_{n}$ is a continuous and differentiable function of $g$ and $\lambda$. The pure Lagrangian multiplier as well as the perturbed Lagrangian methods can be obtained as particular cases of the constraint function method as shown in Pantuso et al. (2000).

Similarly, the tangential frictional condition can be replaced by

$$
w_{s}(v, \tau)=0
$$

where $w_{\mathrm{s}}$ is a continuous and differentiable function of $v$ and $\tau$. More details on the form of the normal and tangential constraint functions can be found in Bathe (1996) and ADINA R\&D, Inc. (2002).

This approach results in the following additional constraint equation for each contact region

$$
\int_{\Gamma_{\mathrm{C}}}\left[w_{n}(g, \lambda) \delta \lambda+w_{s}(v, \tau) \delta \tau\right] \mathrm{d} \Gamma_{\mathrm{C}}=0
$$

\section{Finite element discretization}

Employing standard finite element discretization of Eqs. (7) and (11), we obtain

$$
\mathbf{F}_{u}=\mathbf{R}-\mathbf{R}_{\mathrm{c}}, \quad \mathbf{F}_{\mathrm{c}}=\mathbf{0}
$$

where $\mathbf{F}_{u}$ lists the nodal forces equivalent to the element stresses, $\mathbf{R}$ lists the externally applied loads (including the inertia forces), $\mathbf{R}_{\mathrm{c}}$ represents the contact forces, and $\mathbf{F}_{\mathrm{c}}$ represents the contact constraints of Eq. (11). This nonlinear system of equations is solved incrementally using load (time) incrementation and the Newton-Raphson procedure, in which at each iteration we solve the following system of equations

$$
\left[\begin{array}{cc}
\partial_{\mathbf{U}} \mathbf{F}_{u}+\partial_{\mathbf{U}} \mathbf{R}_{\mathrm{c}} & \partial_{\boldsymbol{\Sigma}} \mathbf{R}_{\mathrm{c}} \\
\partial_{\mathbf{U}} \mathbf{F}_{\mathrm{c}} & \partial_{\boldsymbol{\Sigma}} \mathbf{F}_{\mathrm{c}}
\end{array}\right]^{(i-1)}\left\{\begin{array}{c}
\Delta \mathbf{U} \\
\Delta \boldsymbol{\Sigma}
\end{array}\right\}^{(i)}=\left\{\begin{array}{c}
\mathbf{R}-\mathbf{F}_{u}-\mathbf{R}_{\mathrm{c}} \\
-\mathbf{F}_{\mathrm{c}}
\end{array}\right\}^{(i-1)}
$$

where $\mathbf{U}$ is the assembled displacement vector, and $\mathbf{\Sigma}$ lists the assembled contact variables at each contactor node $k$

$$
\boldsymbol{\Sigma}^{k}=\{\lambda, \tau\}^{k}
$$

A detailed evaluation of the different terms in Eq. (13) can be found in Bathe (1996) and Pantuso et al. (2000). 


\section{Reliability}

For a finite element solution to be reliable, efficient and accurate for engineering design problems, it must be based on reliable formulations and use effective elements that - if possible have been proven to be optimal. In addition, if the design problems involve contact conditions, a reliable contact formulation should be used.

\subsection{General Reliability CONCERns}

Using an appropriate finite element formulation is an essential part of overall solution reliability. Steady state or low/medium speed simulations, for example, should always be treated using implicit analysis. Explicit solutions should only be used in high-speed simulations. For plasticity, it is more effective to use a total strain formulation such as the updated Lagrangian Hencky formulation, rather than a strain rate-type formulation such as the Jaumann formulation (Bathe, 1996). Larger solution steps can be used without introducing additional numerical integration errors.

The 'best' elements for engineering analysis are elements that are not based on numerical factors and satisfy the ellipticity and optimal convergence requirements. This will guarantee that they do not lock, are free of spurious modes, and are robust and maintain their optimal predictive capability (Brezzi and Fortin, 1991; Bathe 1996, 2001a; Chapelle and Bathe, 2003). Elements based on reduced and/or selectively reduced integration, while still used, do not necessarily pass these robustness and reliability requirements.

The ellipticity condition can be expressed as

$$
a\left(\mathbf{v}_{h}, \mathbf{v}_{h}\right) \geq \alpha\left\|\mathbf{v}_{h}\right\|_{1}^{2} \quad \forall \mathbf{v}_{h} \in V_{h}
$$

where $a(.,$.$) is the applicable bilinear form, V_{h}$ denotes the space of finite element functions in the mesh of generic element size $h$, and $\alpha$ is a constant strictly greater than zero (Bathe, 1996). This condition is satisfied when appropriate finite elements and boundary conditions are used. Optimal predictive capability of an element formulation is observed if the following relationship holds

$$
\left\|\mathbf{u}-\mathbf{u}_{h}\right\|_{1} \cong c h^{k}
$$

where $\mathbf{u}$ is the exact solution, $\mathbf{u}_{h}$ is the finite element solution, $k$ is the order of the complete polynomial of the finite elements used and $c$ is a constant that is independent of $h$, independent of critical material properties (such as Poisson's ratio in almost incompressible analysis), and independent of critical geometric features (such as the thickness of a plate or shell).

\subsection{ReLIABILITY IN ANALYSIS OF INCOMPRESSIBLE MEDIA}

Reliable analysis of incompressible and almost incompressible media (like rubber and elastoplastic continua) cannot be performed with displacement-based formulations. Mixed formulations that satisfy ellipticity and the following inf-sup condition are most effective

$$
\inf _{q_{h} \in Q_{h}} \sup _{\mathbf{v}_{h} \in V_{h}} \frac{\left(q_{h}, \operatorname{div} \mathbf{v}_{h}\right)}{\left\|q_{h}\right\|_{0}\left\|\mathbf{v}_{h}\right\|_{1}}=\beta_{h} \geq \beta>0
$$


where $Q_{h}$ is the finite element pressure interpolation space, $V_{h}$ is the displacement interpolation space, and the constant $\beta$ is independent of the mesh size $h$. If an element discretization satisfies this inf-sup condition as well as the ellipticity condition it will be stable and will show optimal convergence behavior. The $\mathrm{u} / \mathrm{p}$ mixed formulation described in (Bathe, 1996) satisfies the above conditions with several displacement/pressure interpolation choices, such as for the $9 / 3$ and $9 / 4-$ c 2D elements and the 27/4 3D element.

\subsection{RELIABILITY IN ANALYSIS OF SHELLS}

Pure displacement-based formulations are also not reliable for plate and shell analyses. They are too stiff (due to locking). Mixed interpolated formulations are much more effective, and are formulated with the aim to satisfy the ellipticity and relevant inf-sup condition. The MITC shell elements have been numerically analyzed and shown to be effective (Bathe et al., 2003; Chapelle and Bathe, 2003; Hiller and Bathe, 2003).

\subsection{RELIABILITY IN ANALYSIS OF CONTACT CONDITIONS}

Reliable contact formulations (like other constrained mixed formulations) must also satisfy an appropriate inf-sup condition (Bathe and Brezzi, 2001; Brezzi and Fortin, 1991)

$$
\sup _{\mathbf{v}_{h} \in V_{h}} \frac{\int_{\Gamma_{\mathrm{C}}} \lambda_{h} g\left(\mathbf{v}_{h}\right) \mathrm{d} \Gamma_{\mathrm{C}}}{\left\|\mathbf{v}_{h}\right\|_{1}} \geq \beta \sup _{\mathbf{v} \in V} \frac{\int_{\Gamma_{\mathrm{C}}} \lambda_{h} g(\mathbf{v}) \mathrm{d} \Gamma_{\mathrm{C}}}{\|\mathbf{v}\|_{1}} \quad \forall \lambda_{h} \in M_{h}
$$

where $M_{h}$ is the space of contact tractions and $\beta$ is a constant, greater than zero. This condition has been evaluated for different contact formulations (Bathe and Brezzi, 2001; El-Abbasi and Bathe, 2001). Commonly used formulations that do not satisfy this condition are the doublepass master slave algorithm, and some master-slave algorithms where the integration is performed at Gaussian points.

Another factor contributing to reliability in contact analysis is the accurate evaluation of the consistent contact stiffness matrix resulting from the different terms of Eq. (13). One commonly overlooked term is that resulting from the change of direction of the contact normal force. This term is proportional to the contact force and the change in the direction of the normal contact vector. It helps maintain a quadratic convergence rate (in the neighborhood of the solution) as the magnitude of the contact force increases.

Finally, ideally, a contact algorithm satisfies the contact patch test (Crisfield, 2000; El-Abbasi and Bathe, 2001; Irons and Razzaque, 1972). In such tests two contacting bodies are loaded in such a way that constant contact tractions should be predicted (see Figure 2). A contact algorithm passes this test if its numerical integration of the contact terms of Eq. (7) in fact results in constant tractions regardless of the meshes/discretizations used for the two contacting bodies. Various contact algorithms in use do not pass the contact patch test (Wriggers, 2002). Node-to-segment contact algorithms, for example, do not pass the patch test when the meshes on both contacting surfaces are different.

A contact algorithm that satisfies the contact inf-sup condition and passes the contact patch test is detailed in (El-Abbasi and Bathe, 2001). In this algorithm, contact is enforced at the nodes of the contactor surface but the integration of Eqs. (7) and (11) accounts for the discontinuous nature of the gap function. 


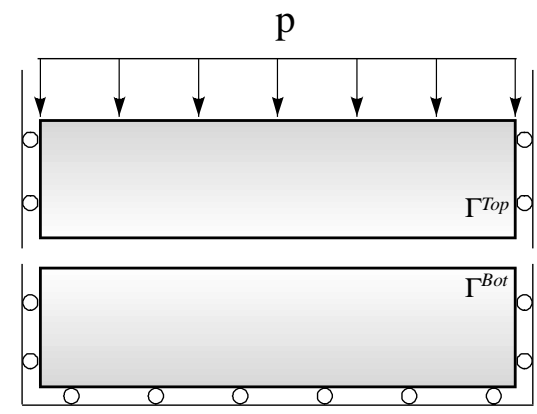

Figure 2. Contact patch test.

(a)
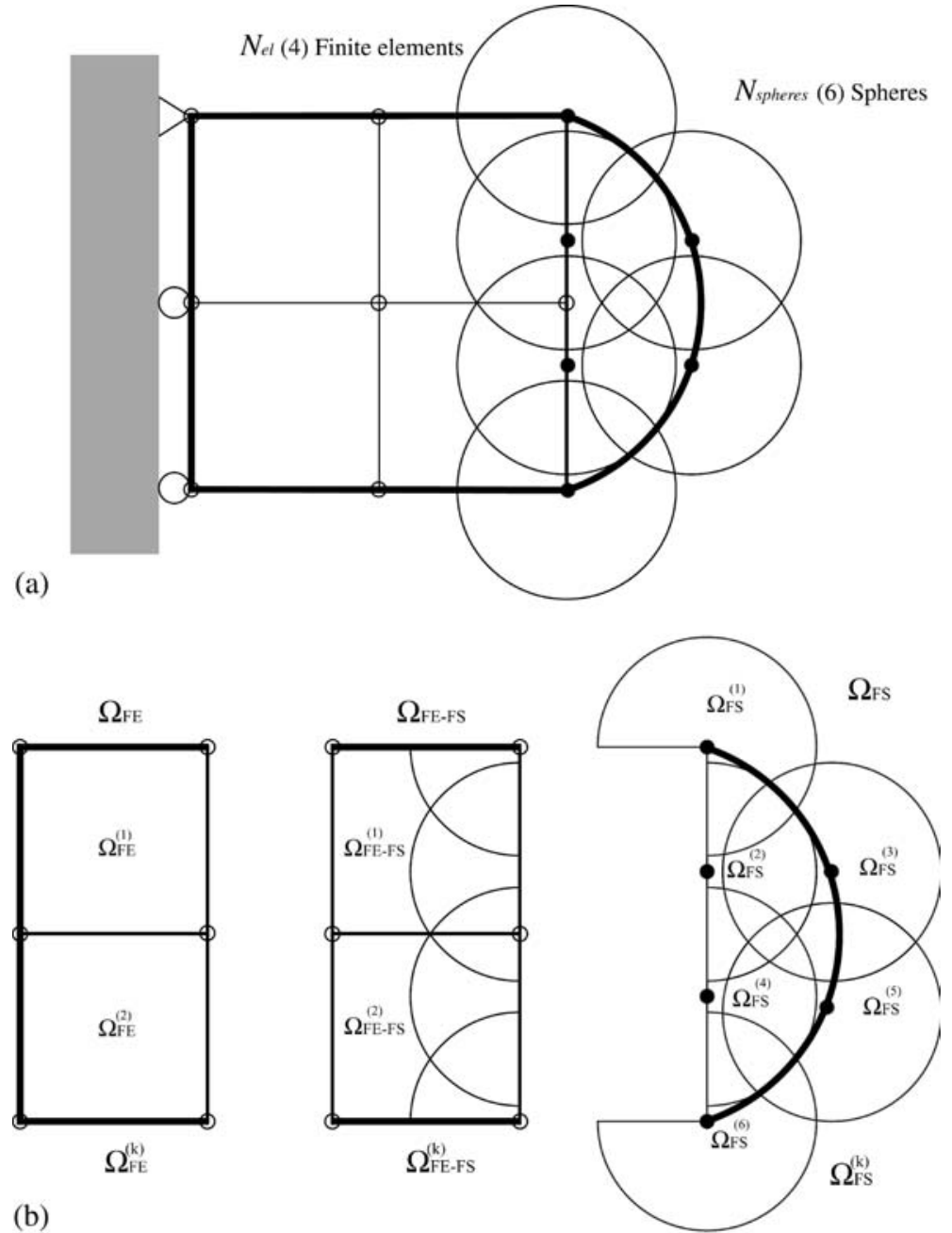

(b)

Figure 3. Coupling finite element discretized domain with finite spheres discretized domain: (a) structure and domains and (b) computational domains. 


\section{Finite spheres coupled with finite element discretizations}

Frequently in finite element analysis, the meshing of a certain part can be difficult because sliver elements need to be avoided. Also, in large deformation analysis or fluid structure interaction (FSI) problems involving contact, sliver elements or highly distorted elements may develop. As well known, highly distorted or sliver elements can lead to unreliable solutions.

To avoid these difficulties and to complement the finite element method, we developed a meshless technique, the method of finite spheres (De and Bathe, 2000, 2001a,b; De et al., 2003; Hong and Bathe, 2003). In meshless techniques including the method of finite spheres, numerical integration is an expensive task. To improve the efficiency of the method of finite spheres, we derived more effective integral equations based on the compact support characteristics of the shape functions (Hong and Bathe, 2003).

The new aspect of the procedure is that it can be used in a consistent manner with the classical finite element method, that is, the coupling of these two procedures satisfies the partition of unity. Also, the method can be used with displacement-pressure interpolations to satisfy the infsup condition (De and Bathe, 2001a). Figure 3 shows a typical discretization using finite elements, finite spheres and a coupling region. We call $\Omega_{\mathrm{FE}}$ the domain which does not have any overlapping with finite spheres, $\Omega_{\mathrm{FE}-\mathrm{FS}}$ the union of finite elements which have non-zero overlap with spheres, and $\Omega_{\mathrm{FS}}$ the region which consists of spheres.

The nodal coefficient vector of values corresponding to the degrees of freedom of the finite elements and finite spheres are

$$
\mathbf{U}^{\mathrm{T}}=\left[\mathbf{u}_{1}^{T} \mathbf{u}_{2}^{T} \cdots \mathbf{u}_{N_{\mathrm{fe}}}^{T} \mid \boldsymbol{\alpha}_{10}^{T} \boldsymbol{\alpha}_{11}^{T} \cdots \boldsymbol{\alpha}_{N_{\text {spheres }}^{T} N_{\mathrm{pol}}}\right]
$$

where the coefficients $\mathbf{u}_{I}^{T}=\left[u_{I} v_{I}\right]$ are the nodal degrees of freedom for finite element node $I$, and $N_{\text {fe }}$ is the number of finite element nodes. Regarding finite spheres, $\boldsymbol{\alpha}_{I m}^{T}=\left[u_{I m} v_{I m}\right]$ are the $m$ th order degrees of freedom at finite sphere node $I, N_{\text {spheres }}$ is the number of finite spheres, and $N_{\text {pol }}$ is the number of terms in the polynomials of the spheres used. For the pure finite element and finite sphere domains, the governing equations are as given in (Bathe, 1996; De and Bathe, 2001b). In the coupled domain we can express the displacement and strain fields as (Hong and Bathe, 2004)

$$
\begin{aligned}
& \mathbf{u}_{\Omega_{\mathrm{FE}-\mathrm{FS}}}(\mathbf{x})=\sum \mathbf{H}_{I}^{\mathrm{FE}-\mathrm{FS}}(\mathbf{x}) \mathbf{u}_{I}+\sum_{J=1}^{N_{\text {spheres }}} \sum_{n \in \Psi} \mathbf{H}_{J n}^{\mathrm{FE}-\mathrm{FS}}(\mathbf{x}) \boldsymbol{\alpha}_{J n} \\
& \boldsymbol{\varepsilon}_{\Omega_{\mathrm{FE}-\mathrm{FS}}}(\mathbf{x})=\sum \mathbf{B}_{I}^{\mathrm{FE}-\mathrm{FS}}(\mathbf{x}) \mathbf{u}_{I}+\sum_{J=1}^{N_{\text {spheres }}} \sum_{n \in \Psi} \mathbf{B}_{J n}^{\mathrm{FE}-\mathrm{FS}}(\mathbf{x}) \boldsymbol{\alpha}_{J n}
\end{aligned}
$$

Here $\mathbf{H}_{I}^{\mathrm{FE}-\mathrm{FS}}(\mathbf{x})$ and $\mathbf{B}_{I}^{\mathrm{FE}-\mathrm{FS}}(\mathbf{x})$ are the displacement matrices and the strain-displacement matrices corresponding to the finite element nodes, respectively. Similarly, $\mathbf{H}_{J n}^{\mathrm{FE}-\mathrm{FS}}(\mathbf{x})$ and $\mathbf{B}_{J n}^{\mathrm{FE}-\mathrm{FS}}(\mathbf{x})$ correspond to the nodes of the finite spheres. The basic equations for the displacement-based coupled discretization using finite elements and finite spheres are

$$
\begin{aligned}
& \sum_{J \in L} \mathbf{K}_{I J} \mathbf{u}_{J}+\sum_{J \in K} \sum_{n \in \Psi} \mathbf{K}_{I J n} \boldsymbol{\alpha}_{J n}=\mathbf{f}_{I}+\hat{\mathbf{f}}_{I} \\
& \sum_{J \in L} \mathbf{K}_{I m J} \mathbf{u}_{J}+\sum_{J \in K} \sum_{n \in \Psi} \mathbf{K}_{I m J n} \boldsymbol{\alpha}_{J n}=\mathbf{f}_{I m}+\hat{\mathbf{f}}_{I m}
\end{aligned}
$$




\section{$10 \quad$ N. Elabbasi et al.}

where Eq. (22) is for node $I$ of finite elements and Eq. (23) for node $I$ of finite spheres. The vectors on the right-hand side incorporate the applied forces, Dirichlet and Neumann boundary conditions (De and Bathe, 2001a,b; Hong and Bathe, 2004).

\section{Numerical examples}

In this section we present some demonstrative solutions obtained using reliable finite element procedures involving contact conditions.

\subsection{Metal Forming: SWAGing Simulation}

The metal forming industry makes extensive use of nonlinear finite element analysis with contact. The workpiece to be shaped usually comes in contact with one or more forming dies, and possibly with itself or with other workpieces. Friction, dynamic and thermal effects should sometimes be taken into consideration. The simulation is always nonlinear due to the plastic deformation of the workpiece and usually large deformations are involved. Due to the above complexities it is essential to use a reliable finite element formulation.

In this example, we simulate a swaging process where a metal tube is reduced in diameter by a series of rapid blows applied through three rigid dies. Figure 4 shows the geometry of the workpiece and the dies, and it also shows the plastic strain at an intermediate stage in the simulation.

\subsection{RUBBER SEAL COMPRESSION}

This example illustrates the importance of the consistent linearization of the contact constraints. It involves the compression of a rubber seal by rigid dies as shown in Figure 5. The rubber seal

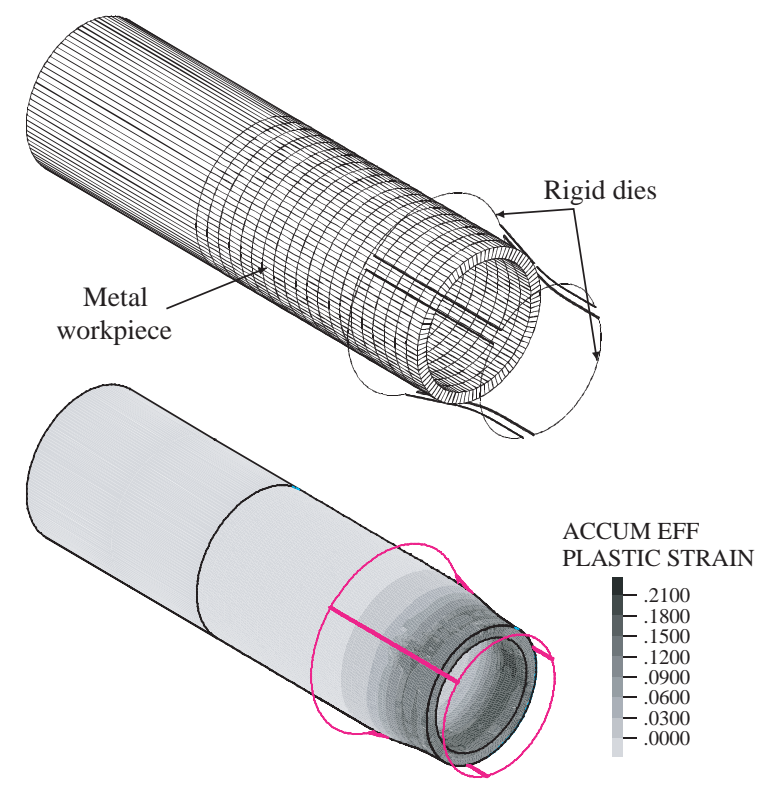

Figure 4. Swaging simulation: finite element mesh (top) and effective strain distribution (bottom). 

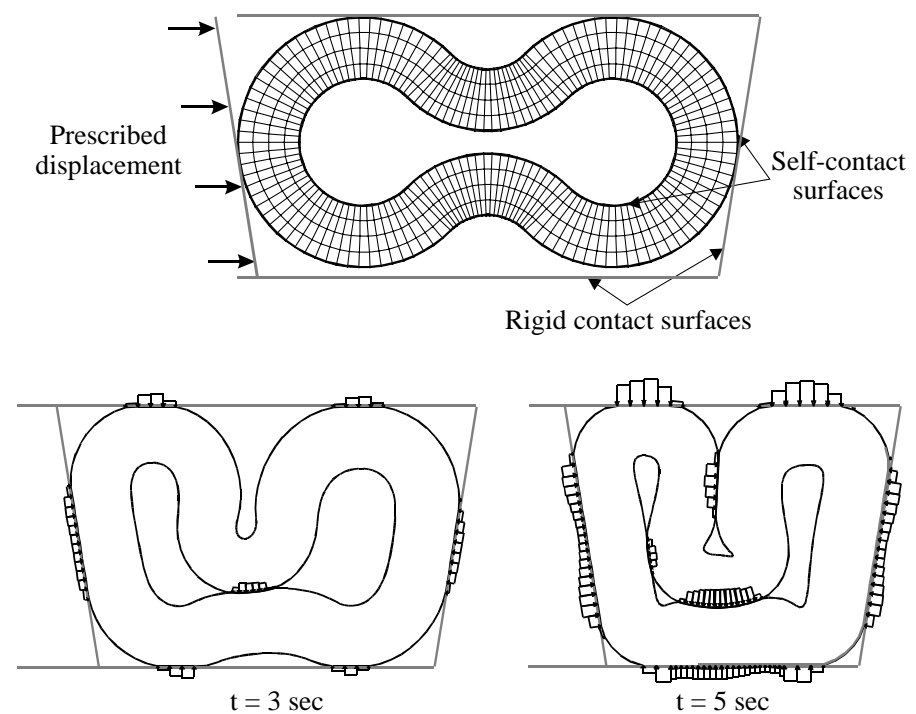

Figure 5. Rubber seal model: schematic (top) and contact pressure distribution at $t=3$ and $5 \mathrm{~s}$ (bottom).

will eventually fold onto itself and experience self-contact where the direction of the contact normal changes as the load increases. Figure 5 also shows the deformed geometry and contact tractions at times $t=3$ and $5 \mathrm{~s}$.

Without the contact stiffness terms mentioned above resulting from Eq. (13), the simulation does not converge at time $t=3 \mathrm{~s}$, that is beyond time $t=2 \mathrm{~s}$ (at $t=2$ the rubber folds upon itself). Figure 6 shows the out-of-balance energy norm as a function of the iterations with and without the above mentioned consistent contact stiffness terms. The results indicate the loss of quadratic convergence when the extra terms are neglected.

These stiffness terms also affect the natural frequencies calculated based on the deformed configuration (contact is accounted for in the frequency analysis). Table 1 shows the first five natural frequencies at time $t=2 \mathrm{~s}$ with and without the extra terms. In this case, the effect of the extra stiffness term is rather small.

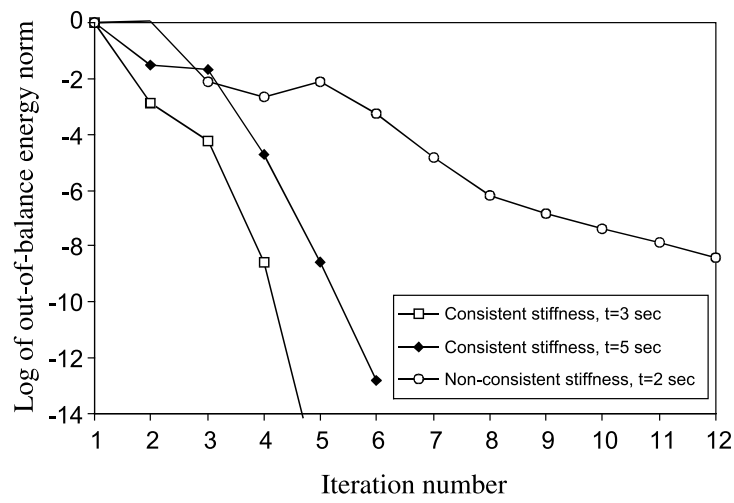

Figure 6. Energy convergence tolerance during rubber seal compression. 


\section{$12 N$. Elabbasi et al.}

Table 1. Effect of extra stiffness terms on the first five natural frequencies of the rubber seal at time $t=2 \mathrm{~s}$

\begin{tabular}{llllll}
\hline \multicolumn{5}{l}{ Natural frequencies } \\
\cline { 2 - 6 } Without extra terms & 154.4 & 203.2 & 440.1 & 636.3 & 688.3 \\
With extra terms & 154.5 & 208.7 & 440.6 & 645.0 & 690.8 \\
\hline
\end{tabular}

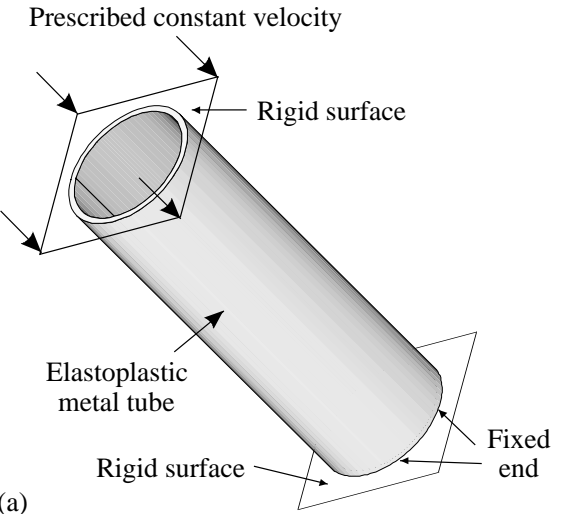

(b)

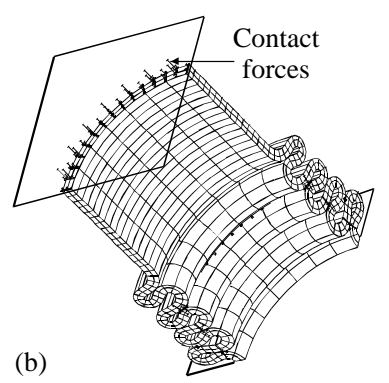

Figure 7. Tube compression: (a) schematic and (b) deformed geometry and contact forces.

\subsection{TubE COMPRESSION}

In this example, a $10 \mathrm{~cm}$ long cylindrical steel tube is fixed at one end and compressed at the other end through contact with a rigid surface having a prescribed constant velocity, as shown in Figure 7(a). This example involves self-contact (surfaces contacting themselves) along the inner and outer walls of the tube and contact with rigid surfaces at both ends of the tube. Figure 7(b) shows the deformed geometry, while Figure 8 shows the load-deflection curve when the tube is compressed at speeds of 0.1 and $10 \mathrm{~m} / \mathrm{s}$.

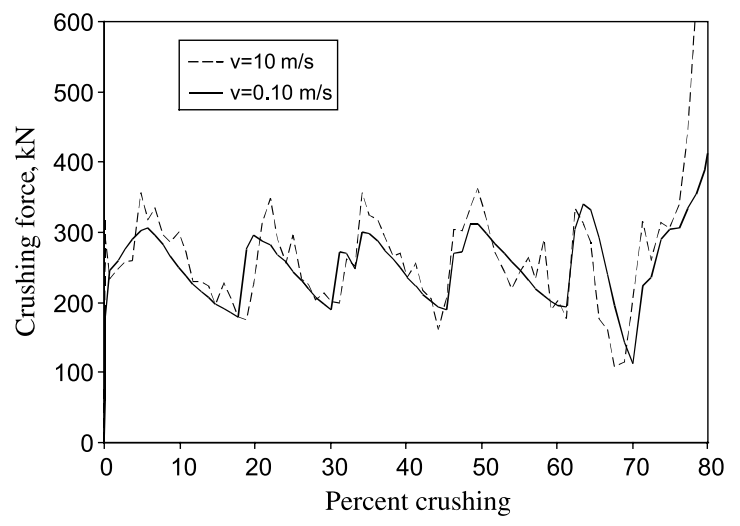

Figure 8. Load deflection curve for tube compression for two different crushing velocities. 


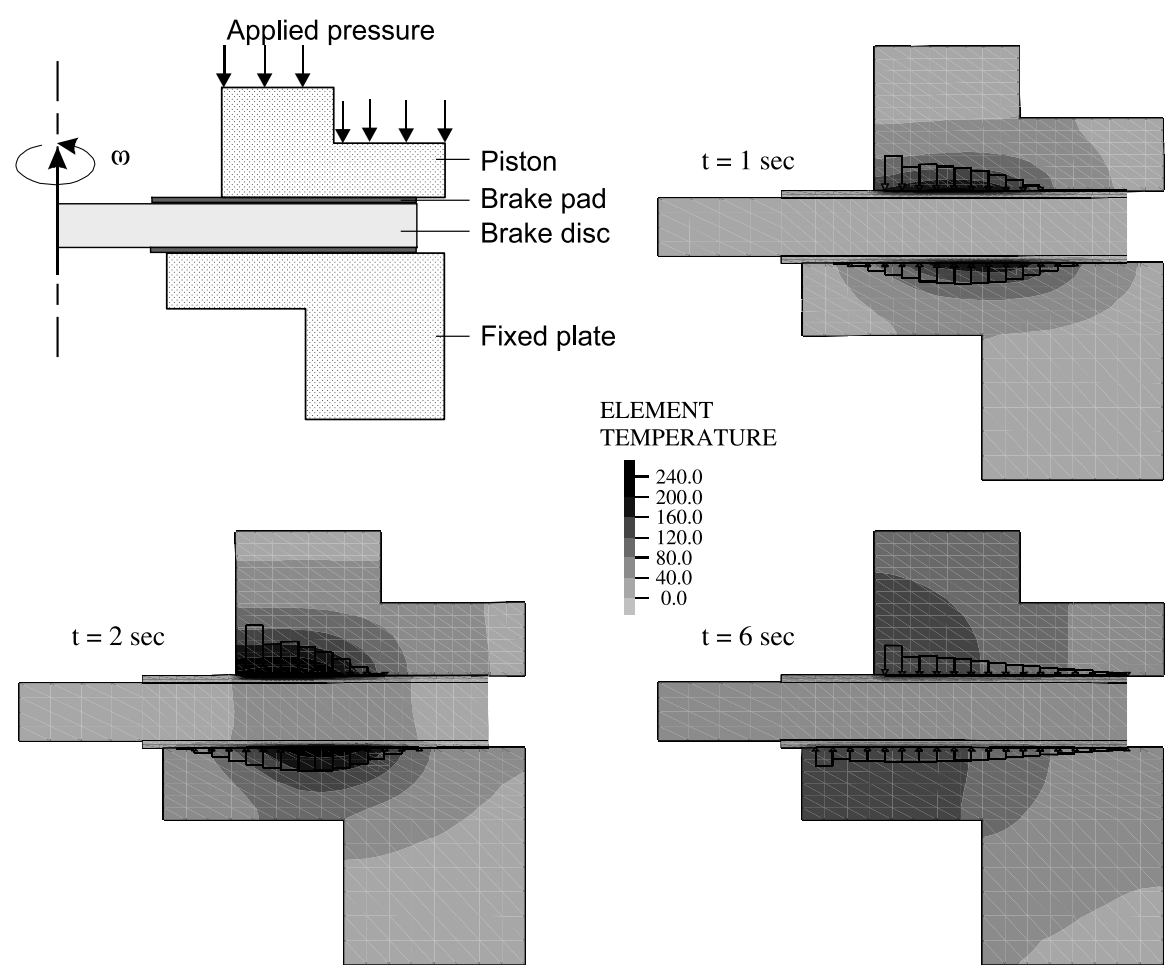

Figure 9. Disc braking system: schematic (top left) and temperature and contact pressure distribution at $t=1,2$ and $6 \mathrm{~s}$.

\subsection{BRAKE SIMULATION: THERMOMECHANICAL COUPLING}

This example involves a thermally coupled disc brake analysis. In Figure 9 we show a schematic of the disc braking system where the heat generation caused by friction between the rotating disc and the piston and plate is to be analyzed. In this simulation, the rotating disc is prescribed to slow down from 300 to $0 \mathrm{rpm}$ in $5 \mathrm{~s}$. The analysis is performed using a simple 2-D axisymmetric model subjected to an out-of-plane contact slipping which is the main source of heat generation. Figure 9 also shows the temperature distribution on the brake components at times $t=1,2$ and $6 \mathrm{~s}$.

\subsection{BiOMEDICAL APPLICATIONS: AORTIC VALVE SIMULATION}

During recent years there has been an increasing interest in the numerical solution of biomedical problems (Bathe, 2001b, 2003). Contact plays a major role in many of these problems, especially in the areas of hemodynamics, orthotics, prosthetics and crash dynamics. In many of the problems analyzed, experimental data are hard to obtain, and the analyst should therefore use reliable numerical tools. In this example, we simulate blood flow through an aortic valve. This coupled multiphysics problem involves FSI as well as contact effects.

Figure 10 shows the fluid and solid meshes of a simplified heart valve geometry. The artery was modeled as a hyperelastic solid and was meshed with nine node $\mathrm{u} / \mathrm{p}$ elements. The blood was modeled as a Newtonian fluid and was meshed with four node elements. Note that the solid 

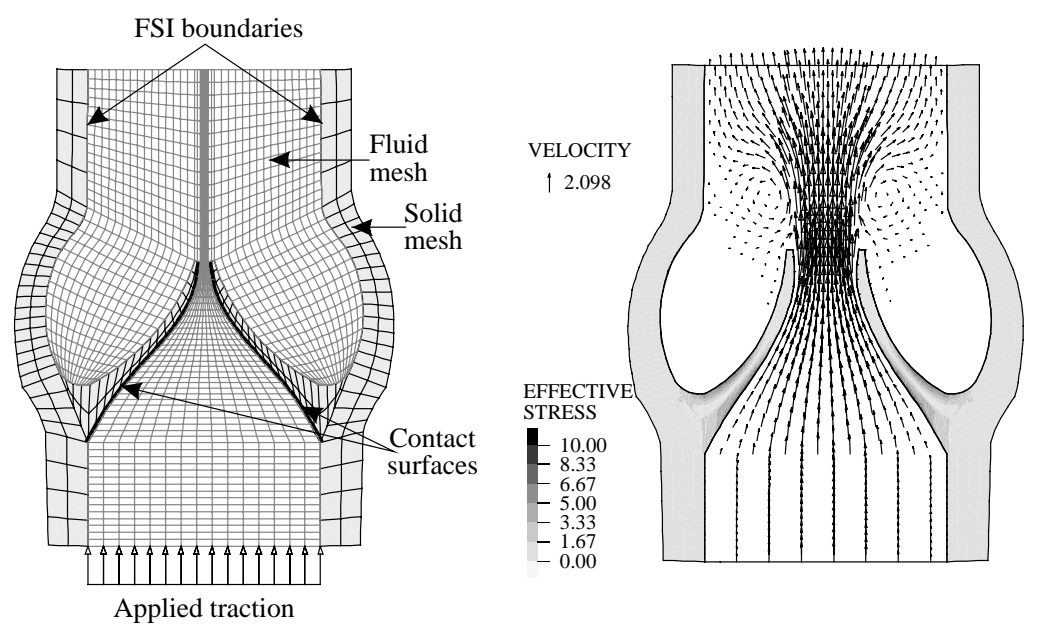

Figure 10. Schematic of aortic valve (left) and results during valve opening showing velocity vector plot in the blood and effective stress in the artery (right).

and fluid meshes are non-matching at the FSI boundary. Figure 10 also shows the velocity vector plot in the fluid and the effective stress in the solid as the valve is opening.

\subsection{ANALysis of A PLATE WITH A HOLE USING FINITE SPHERES}

We consider a square plate with a small circular hole. The plate is subjected to a uniform pressure as shown in Figure 11. To simulate the stress concentration phenomenon, usually, in the traditional finite element solution, mesh refinements are used around the hole. In our analysis, we used rather coarse almost uniform finite element meshes enriched with only two finite spheres, with $N_{\mathrm{pol}}=3$, placed as shown in Figure 11. Table 2 shows the normal stress in the horizontal direction, $\sigma_{x x}$, for different finite element mesh densities. The results show that

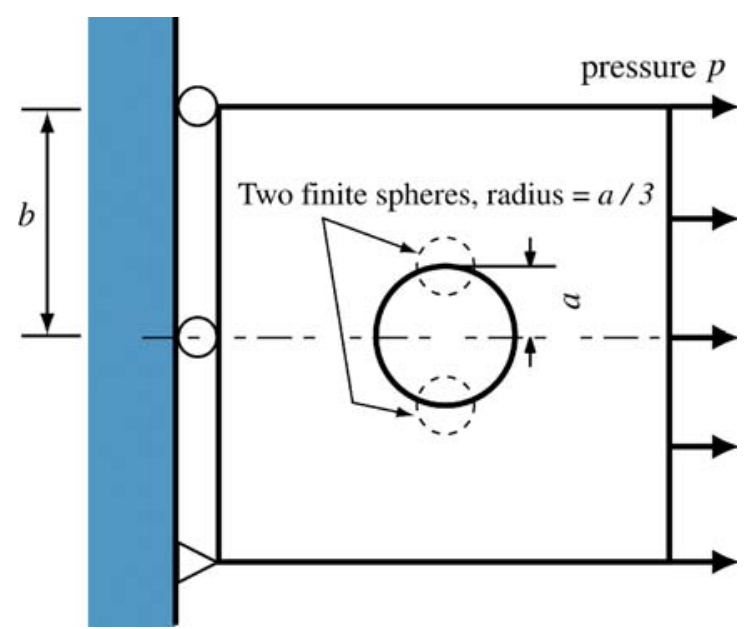

Figure 11. Geometry of a square plate with a hole in the middle of the plate. Young's modulus $\mathrm{E}=100$, Poisson's ratio $=0.30, a / b=0.10, p=1.0$. 
Table 2. Comparison of maximum stress value in the plate with a hole shown in figure 11

\begin{tabular}{lllll}
\hline $\begin{array}{l}\text { No. of elements } \\
\text { (full plate) }\end{array}$ & FEM (4-nodes) & FEM (9-nodes) & Enriching scheme & Accurate value \\
\hline 16 & 1.455 & 1.937 & 2.464 & 3.08 \\
64 & 1.878 & 2.388 & 2.649 & 3.08 \\
256 & 2.383 & 2.785 & 2.957 & 3.08 \\
\hline
\end{tabular}

the two added finite spheres lead to a significant improvement in stress prediction, especially when using 4-node finite elements. Since lower-order finite elements are in general attractive for the solution of contact problems, clearly, the potential of using finite spheres in conjunction with finite elements is significant.

\section{Concluding remarks}

In this paper we focused on the reliability of solution needed for the accurate analysis of engineering design problems involving contact. A solution procedure for frictional contact problems based on the constraint function method was summarized, and the reliability of different aspects of finite element solutions was discussed. It can be concluded that reliability is an important aspect of any finite element analysis in order to avoid erroneous results. Today, reliable finite element solutions can be obtained for many complex engineering problems, and we presented several demonstrative applications in Section 6. Also, new discretization techniques, such as the method of finite spheres mentioned in this paper, can be expected to further increase the reliability and effectiveness of engineering analysis.

\section{References}

ADINA R\&D, Inc. (2002). ADINA Theory and Modeling Guide. Volumes I, II, III, Watertown, MA.

Bathe, K.J. (1996). Finite Element Procedures. Prentice Hall, New Jersey.

Bathe, K.J. (2001a). The inf-sup condition and its evaluation for mixed finite element methods. Computers and Structures 79, 243-252, 971.

Bathe, K.J. (ed.) (2001b). Computational Fluid and Solid Mechanics. Proceedings of the First MIT Conference on Computational Fluid and Solid Mechanics. Elsevier Science.

Bathe, K.J. (ed.) (2003). Computational Fluid and Solid Mechanics 2003. Proceedings of the Second MIT Conference on Computational Fluid and Solid Mechanics. Elsevier Science.

Bathe, K.J. and Brezzi, F. (2001). Stability of finite element mixed interpolations for contact problems. Proceedings of the National Academy of Lincei 12, 159-166.

Bathe, K.J., Lee, P.S. and Hiller, J.F. (2003). Towards improving the MITC9 shell element. Computers and Structures 81, 477-489.

Brezzi, F. and Fortin, M. (1991). Mixed and Hybrid Finite Element Methods, Springer.

Chapelle, D. and Bathe, K.J. (2003). The Finite Element Analysis of Shells-Fundamentals, Springer.

Crisfield, M.A. (2000). Re-visiting the contact patch test. International Journal for Numerical Methods in Engineering 48, 435-449.

De, S. and Bathe, K.J. (2000). The method of finite spheres. Computational Mechanics 25, 329-345.

De, S. and Bathe, K.J. (2001a). Displacement/pressure mixed interpolation in the method of finite spheres. International Journal for Numerical Methods in Engineering 51, 275-292.

De, S. and Bathe, K.J. (2001b). The method of finite spheres with improved numerical integration. Computers and Structures 79, 2183-2196.

De, S., Hong, J.W. and Bathe, K.J. (2003). On the method of finite spheres in applications: towards the use with ADINA and in a surgical simulator. Computational Mechanics 31(1-2), 27-37. 


\section{$16 \quad$ N. Elabbasi et al.}

El-Abbasi, N. and Bathe, K.J. (2001). Stability and patch test performance of contact discretizations and a new solution algorithm. Computers and Structures 79, 1473-1486.

Eterovic, A.L. and Bathe, K.J. (1991). On the treatment of inequality constraints arising from contact conditions in finite element analysis. Computers and Structures 40, 203-209.

Hiller, J.F. and Bathe, K.J. (2003). Measuring convergence of mixed finite element discretizations: An application to shell structures. Computers and Structures 81, 639-654.

Hong, J.W. and Bathe, K.J. (2003). On analytical transformations for efficiency improvements in the method of finite spheres. In K.J. Bathe (ed.) Computational Fluid and Solid Mechanics. Elsevier.

Hong, J.W. and Bathe, K.J. (2004). Coupling and enrichment schemes for finite element and finite sphere discretizations. Computers and Structures, to appear.

Irons, B.M. and Razzaque A. (1972). Experience with the patch test for convergence in finite elements. In A.K. Aziz (ed.) The Mathematical Foundations of the Finite Element Method with Applications to Partial Differential Equations. Academic Press, New York, pp. 557-587.

Pantuso, D., Bathe, K.J. and Bouzinov, P.A. (2000). A finite element procedure for the analysis of thermomechanical solids in contact. Computers and Structures 75, 551-573.

Wriggers, P. (2002). Computational Contact Mechanics. Wiley, Hoboken, NY, 441 pp. 